\title{
Superheated steam pretreatment of cellulose affects its electrospinnability for microfibrillated cellulose production
}

\begin{abstract}
In this study, oil palm mesocarp fiber (OPMF) was pretreated with (1) superheated steam (SHS) and (2) potassium hydroxide $(\mathrm{KOH})$ to remove hemicellulose. Both SHS-OPMF and $\mathrm{KOH}-$ OPMF underwent delignification step to isolate the cellulose and dissolved in selected ionic liquid and its co-solvent before being electrospun to obtain microfibrillated cellulose (MFC). FE-SEM images showed that SHS-OPMF cellulose produced discontinuous MFC fiber with diameter ranging from 100 to $500 \mathrm{~nm}$, of which $15.5 \%$ were in the range of 100-200 nm; while $\mathrm{KOH}-\mathrm{OPMF}$ cellulose produced continuous MFC with sizes larger than $200 \mathrm{~nm}$. The differences in fiber size and continuity of fiber produced were due to incomplete removal of hemicellulose from SHS-OPMF sample that inhibited fiber re-coalescence and resulted in interruption in fiber formation.
\end{abstract}

Keyword: Microfibrillated cellulose; Hemicellulose; Superheated steam; Electrospinning 